\title{
Índices de sensibilidade à insulina em fêmeas caninas: efeito do ciclo estral e da piometra
}

\author{
Insulin sensitivity indexes in female dogs: \\ effect of estrus cycle and pyometra
}

\section{Álan Gomes Pöppl ${ }^{1,3}$, Camila Serina Lasta ${ }^{1,2}$, Félix Hilário Díaz González², Luiz Carlos Kucharski ${ }^{3}$ \& Roselis Silveira Martins da Silva ${ }^{3}$}

\begin{abstract}
RESUMO
A ocorrência do estro e diestro em fêmeas caninas está associada à presença de resistência à insulina. Além disso, estados sépticos e inflamatórios, como observado frente à piometra, também cursam com resistência à ação insulínica. $\mathrm{O}$ objetivo deste estudo foi verificar qual o efeito das diferentes fases do ciclo estral e da piometra sobre diferentes índices de sensibilidade à insulina. Com base em medidas de glicemia e insulinemia basal em fêmeas caninas em diferentes fases do ciclo estral, e em pacientes com piometra, foram calculados os índices HOMA B, HOMA R, índice insulinogênico, relação insulina:glicose corrigida, determinação da fructosaminemia e relação fructosamina/albumina. $O$ índice insulinogênico e a relação insulina:glicose corrigida mostraram-se mais sensíveis na detecção de hiperinsulinemia relativa, especialmente em pacientes com piometra. A falta de valores de corte para os índices HOMA prejudicou sua avaliação, apesar de terem sido eficazes em demonstrar resistência à insulina e maior secreção pancreática de insulina em fêmeas com piometra. Os diferentes índices de sensibilidade mostraram-se como ferramentas de fácil aplicação, úteis e relativamente pouco onerosas, apresentando vantagens em relação à determinação isolada de glicose e insulina séricas em jejum. A fructosamina não foi eficaz em evidenciar diferenças significativas entre as diferentes fases do ciclo estral, apesar de ter sofrido forte influência da albuminemia nas pacientes com piometra. A determinação da relação fructosamina:albumina é mais indicada do que a avaliação da fructosamina isolada, sendo necessários estudos para determinar valores de referência desta relação, bem como para os índices HOMA B e HOMA R em caninos.
\end{abstract}

Descritores: resistência à insulina, diestro, piometra, relação insulina:glicose.

\begin{abstract}
Ocurrence of estrous cycle and pyometra in female dogs are associated to insulin resistance status. Moreover, septic/ inflammatory environments such as pyometra may also course with reduced insulin sensitivity. The aim of this study was to check the effect of estrus cycle and pyometra on insulin sensitivity indexes. Based in blood basal glucose levels and serum insulin basal levels from bitches in different estrus cycle phases as well as in bitches with pyometra, different insulin sentivity indexes were calculated (HOMA B, HOMA R, insulinogenic index, amended insulin:glucose ratio, fructosamine and fructosamine:albumin ratio). The insulinogenic index and amended insulin:glucose ratio were more sensible in detecting relative hyperinsulinemic status, specially in pyometra patients. The lack of a cut-off point for HOMA indexes impairs its interpretation, notwithstanding their have been able to shown insulin resistance in female dogs with pyometra. Moreover, this different sensitivity indexes have prove to be really easy, cheap and useful and have some advantages comparing with basal insulin and glucose levels. Fructosamine was not a useful screening test in detecting differences in insulin sensitivity status despite there were a strong influence of hypoalbuminemia on fructosamine levels in bitches with pyometra. Fructosamine:albumina ratio is more reliable than fructosaminemia alone, and future studies are need to determine reference values for this ratio, as well to HOMA B and HOMA R indexes in female dogs.
\end{abstract}

Keywords: insulin resistance, diestrus, pyometra, insulin:glucose ratios.

${ }^{1}$ Programa de Pós-graduação em Ciências Veterinárias da Universidade Federal do Rio Grande do Sul (PPGCV-UFRGS), Faculdade de Veterinária, Universidade Federal do Rio Grande do Sul (UFRGS), Av. Bento Gonçalves no. 9090, 91540-000 Porto Alegre, Bairro Agronomia, RS, Brasil. ${ }^{2}$ Laboratório de Análises Clínicas Veterinárias, Faculdade de Veterinária-UFRGS. ${ }^{3}$ Laboratório de Metabolismo e Endocrinologia Comparada, Departamento de Fisiologia, Instituto de Ciências Básicas da Saúde-UFRGS. CORRESPONDÊNCIA: A.G. Pöppl [gomespoppl@hotmail.comFax: + 55 (51) 3308 6112]. 


\section{INTRODUÇÃO}

A diabetes mellitus canina (DMC) é uma das doenças endócrinas mais comuns na clínica de pequenos animais $[17,40]$ e nas últimas décadas observou-se um aumento na prevalência hospitalar de casos deste transtorno [15]. Esta maior incidência de DMC vem acompanhando a tendência de pandemia de diabetes observada em humanos, especialmente associada a fatores diabetogênicos, como o sedentarismo e a obesidade, além de maior preocupação dos proprietários e melhor entendimento da doença pelos veterinários $[4,15,30]$. Apesar da DMC apresentar uma etiopatogênia muito semelhante a da diabetes mellitus do tipo I (DM I) em humanos, com detecção de autoanticorpos anticomponentes das ilhotas pancreáticas $[17,40]$, muitas características da DMC assemelham-se à diabetes mellitus gestacional (DMG) humana $[4,35,40,44]$.

Apesar de poucos trabalhos correlacionarem piometra e diabetes [22,24,37], o complexo da hiperplasia endometrial cística - piometra configura um estado séptico e inflamatório crônico [1,12,48], e a ocorrência de qualquer desordem séptica ou inflamatória pode causar resistência à insulina $[6,9,14]$. Além disso, recentemente demonstrou-se que a ocorrência do estro, do diestro e da piometra em cães pode prejudicar a atividade tirosina quinase de membrana no tecido muscular, além de reduzir a afinidade da ligação entre a insulina e o seu receptor neste tecido [39]. O objetivo deste estudo é verificar o impacto do ciclo estral e do complexo hiperplasia endometrial cística - piometra sobre diferentes índices de sensibilidade à insulina.

\section{MATERIAIS E MÉTODOS}

Vinte e uma fêmeas caninas foram utilizadas neste estudo, sendo cinco delas em anestro, três em estro, sete em diestro e seis com piometra. As pacientes, nas diferentes fases do ciclo estral, foram selecionadas do Projeto de Controle Reprodutivo do Hospital de Clínicas Veterinárias da Universidade Federal do Rio Grande do Sul, ao passo que as pacientes com diagnóstico de piometra foram selecionadas da rotina de atendimentos. As fases do ciclo estral foram definidas por citologia vaginal e histórico reprodutivo [50]. Como critérios de inclusão no estudo para as pacientes do grupo ciclo estral foram adotados os seguintes parâmetros: hemograma sem alterações [49], condição corporal eutrófica [25], ausência de doenças ou uso concomitante de qualquer tipo de medicação, além de não uso de progestágenos pelo menos nos últimos seis meses. Para as pacientes com piometra, foram adotados os mesmos critérios de inclusão, exceto o hemograma, que, neste caso, deveria apresentar leucocitose como um marcador do estado séptico/inflamatório promovido por esta condição [49]. A participação dos animais no estudo foi autorizada por seus proprietários, por meio de um termo de consentimento informado. Este estudo foi aprovado pelo Comitê de Ética em Pesquisa da Universidade Federal do Rio Grande do Sul (CEP/UFRGS - projeto $\mathrm{n}^{\circ}$ 2006622).

Após jejum de 12 horas, foram coletadas amostras de sangue da veia jugular em tubo com EDTA para determinação de hemograma $(2 \mathrm{~mL})$ e em tubos sem anticoagulante $(3 \mathrm{~mL})$ para determinações bioquímicas. A quantidade total de eritrócitos, leucócitos e hemoglobina foi determinada por diluição do sangue e leitura em contador automático ${ }^{1}$. O hematócrito foi obtido por centrifugação do sangue total em tubo capilar (10.000rpm por 5 minutos). Os valores de VGM e CHGM foram calculados [49]. A leucometria diferencial foi determinada por leitura de esfregaço sanguíneo corado pelo método Panótico rápido. A proteína plasmática total foi determinada por refratometria. A plaquetometria foi determinada a partir de contagem em câmara de Neubauer.

As determinações de glicemia foram realizadas com glicímetro portátil ${ }^{2}$. As amostras coletadas para determinação da concentração de insulina foram centrifugadas a $10.000 \mathrm{rpm}$ por 10 minutos, sendo o soro posteriormente separado e congelado a $-20^{\circ} \mathrm{C}$ até posterior análise. Para determinação dos valores de insulina, foi utilizada a técnica de radioimunoensaio para insulina humana ${ }^{3}$. As amostras foram dosadas em duplicata.

Com base na determinação da insulinemia e glicemia basal, foram calculados quatro índices de sensibilidade à insulina: índice insulinogênico (II $)=$ insulinemia $(\mu \mathrm{U} / \mathrm{mL}) /$ glicemia $(\mathrm{mg} / \mathrm{dL})$; relação insulina:glicose corrigida (RI:GC) $=$ insulinemia $(\mu \mathrm{U} / \mathrm{mL}) \times 100 /$ glicemia $(\mathrm{mg} / \mathrm{dL})-30[9,23]$; HOMA B $(\%-\mathrm{HB})=20 \mathrm{x}$ insulinemia $(\mu \mathrm{U} / \mathrm{mL}) /$ glicemia $(\mathrm{mmol} / \mathrm{L})-3,5$ e o HOMA R $(\mathrm{HR})=$ insulinemia $(\mu \mathrm{U} / \mathrm{mL}) \times$ glicemia $(\mathrm{mmol} / \mathrm{L}) / 22,5$ [28]. Além desses índices, foi realizada a determinação da concentração sérica de fructosamina e de albumina utilizando kits diagnósticos específicos ${ }^{4}$. Quando necessário, foi calculada a relação fructosamina $(\mu \mathrm{mol} / \mathrm{L}) /$ albumina $(\mathrm{g} / \mathrm{L})$. 
Os resultados foram expressos em média e desvio padrão ou mediana e amplitude interquartil, conforme distribuição dos resultados [51]. Para fins de interpretação, as análises foram realizadas comparando-se o grande grupo ciclo estral entre si (anestro como controle, estro e diestro) por meio de análise de variância de uma via (ANOVA One Way), seguido do teste de Tukey. As pacientes com piometra foram comparadas às pacientes em diestro, uma vez que a piometra é uma condição típica desta fase do ciclo estral [48] pelo teste t de Student. Quando necessário, conforme a distribuição, normalidade e variância dos dados, foram aplicados, respectivamente, os testes de Mann-Whitney, e Kruskal-Wallis, em substituição ao teste $\mathrm{t}$ de Student e ANOVA One Way, respectivamente. Também realizou-se análise de correlação de Pearson entre alguns parâmetros, quando pertinente. Para todas as análises, foi utilizado o programa Sigma Stat 2.0 para Windows; considerando-se significativa uma diferença entre médias com $\mathrm{p}<0,05$.

\section{RESULTADOS}

A Tabela 1 apresenta médias, desvio padrão e intervalo de idade, peso e escore corporal das pacientes participantes do estudo nas diferentes fases do ciclo estral.
As pacientes com piometra apresentaram idade e peso significativamente superiores $(\mathrm{p}<0,05)$ às pacientes em diestro. $\mathrm{O}$ valor médio do leucograma das fêmeas com piometra foi de $27,7 \pm 10,2 \times 10^{3} / \mathrm{mm}^{3}(16,5-45,1)$.

Não houve diferença significativa entre as médias de glicemia dos diferentes grupos estudados (anestro $89,1 \pm 10,9 \mathrm{mg} / \mathrm{dL}$; estro $76,4 \pm 8,3 \mathrm{mg} / \mathrm{dL}$; diestro $86,8 \pm 11,1 \mathrm{mg} / \mathrm{dL}$ e piometra $84,9 \pm 12,2 \mathrm{mg} / \mathrm{dL}$; $\mathrm{p}>0,05)$. Nas diferentes fases do ciclo estral, também não foram observadas diferenças significativas nos valores de insulinemia basal (anestro 17,1 $\pm 6,5 \mu \mathrm{U} / \mathrm{mL}$; estro $15,3 \pm 6,7 \mu \mathrm{U} / \mathrm{mL}$; diestro $21 \pm 9,3 \mu \mathrm{U} / \mathrm{mL}$; $\mathrm{p}>0,05)$, contudo a insulinemia basal das paciente com piometra foi significativamente superior $(43,5 \pm$ $21,8 \mu \mathrm{U} / \mathrm{mL} ; \mathrm{p}<0,05)$ às fềmeas em diestro.

A Tabela 2 apresenta os resultados obtidos para o II e RI:GC. Não houve diferença significativa entre as diferentes fases do ciclo estral $(\mathrm{p}>0,05)$. No entanto, as pacientes com piometra apresentaram valores para II e RI:GC superiores $(\mathrm{p}<0,05)$ aos observados nas pacientes em diestro. Quanto aos índices HOMA B e HOMA R observou-se o mesmo perfil em pacientes ao longo do ciclo estral, não se detectando diferenças significativas entre as medianas para estes índices (Figura 1). Para as pacientes com piometra, os

Tabela 1. Média, desvio padrão e intervalo de idade, peso e escore corporal das pacientes avaliadas em diferentes fases do ciclo estral e em fêmeas com piometra.

\begin{tabular}{cccc}
\hline Categoria & Idade (anos) & Peso (kg) & Escore corporal \\
\hline Anestro & $1,32 \pm 0,6(0,5-2)$ & $14,9 \pm 6,19(7,5-29)$ & $2,81 \pm 0,32(2,5-3,5)$ \\
Estro & $2,34 \pm 1,26(0,9-2,5)$ & $15,7 \pm 7,36(6-29)$ & $3,14 \pm 0,24(3-3,5)$ \\
Diestro & $3,65 \pm 3,25(0,7-10)$ & $16,6 \pm 8,5(6,1-26,6)$ & $3,15 \pm 0,31(3-3,5)$ \\
Piometra & $7,12 \pm 3,09(4-13)^{*}$ & $27,7 \pm 11,3(17-53)^{*}$ & $3 \pm 0,26(2,5-3,5)$ \\
\hline
\end{tabular}

*indica diferença significativa $(\mathrm{p}<0,05)$ do grupo piometra em relação ao grupo diestro.

Tabela 2. Média, desvio padrão e intervalo dos valores obtidos para índice insulinogênico e relação insulina:glicose corrigida nas diferentes fases do ciclo estral e em pacientes com piometra.

\begin{tabular}{ccc}
\hline Categoria & Índice Insulinogênico* & Relação Ins:Gli corrigida** \\
\hline Anestro & $0,186 \pm 0,06(0,127-0,262)$ & $27,8 \pm 9,26(21,8-39,2)$ \\
Estro & $0,187 \pm 0,07(0,140-0,271)$ & $29,9 \pm 10,64(23,15-42,2)$ \\
Diestro & $0,250 \pm 0,12(0,131-0,418)$ & $39,1 \pm 22,04(19,37-80,8)$ \\
Piometra & $0,504 \pm 0,24(0,162-0,805)^{\#}$ & $78,35 \pm 36,46(25,09-122,9)^{\#}$ \\
\hline
\end{tabular}

*valor de referência: aumentado $>0,235 ; * *$ valor de referência: aumentado $>30$; ${ }^{*}$ representa diferença significativa $(\mathrm{p}<0,01)$ entre o grupo piometra e o grupo diestro. 

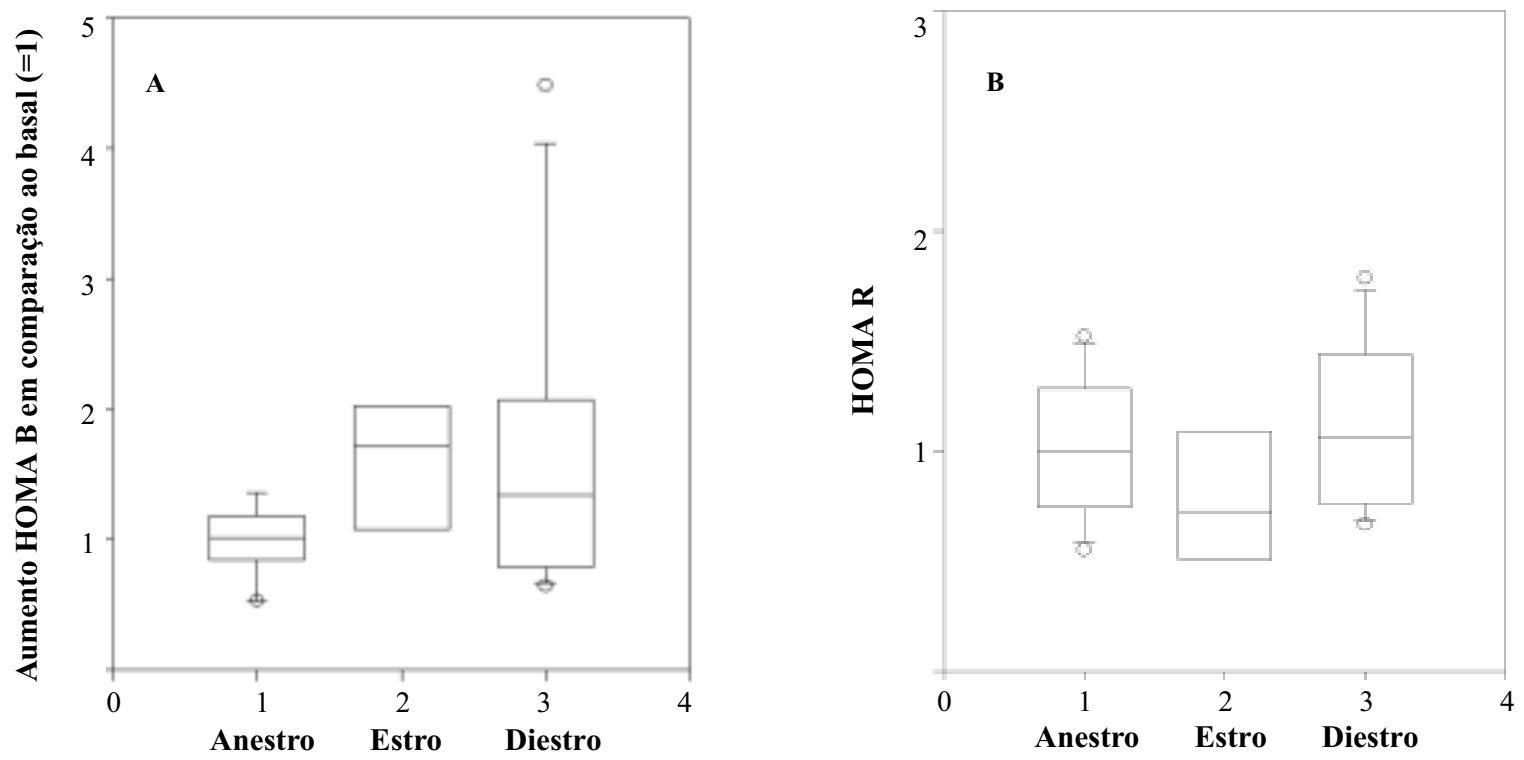

Figura 1. [A] Medianas, amplitude interquartil e percentis 10 e 90\% do aumento no índice HOMA B em relação ao basal (grupo anestro =1). [B] Medianas, amplitude interquartil e percentis 10 e 90\% do índice HOMA R. Não foi detectada diferença significativa pelo teste de Kruskall-Wallis para ambos índices ( $\mathrm{p}>0,05)$.

índices HOMA B e HOMA R mostraram valores significativamente maiores aos observados em pacientes em diestro $(\mathrm{p}<0,05)$, como demonstrado na Figura 2.

Os valores de fructosaminemia nas diferentes fases do ciclo estral não apresentaram diferenças significativas $(p>0,05)$. No entanto, o grupo piometra apresentou valores de fructosaminemia significativamente mais baixos $(p<0,05)$ em comparação ao grupo diestro (Tabela 3), o que esteve associado a valores de albumina também significativamente menores $(\mathrm{p}<0,001)$ no

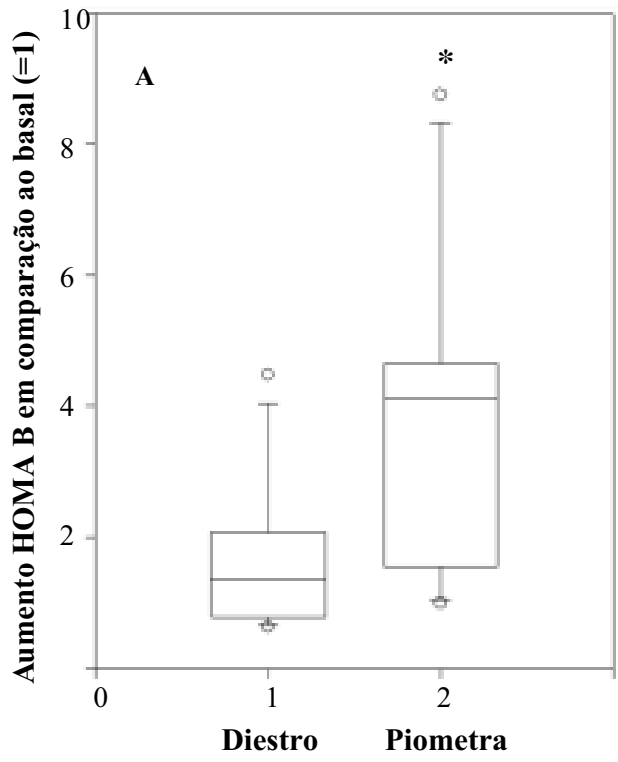

grupo piometra $(18,7 \pm 4,1 \mathrm{~g} / \mathrm{dL})$ em comparação ao grupo diestro $(30,3 \pm 3,7)$. A diferença na relação fructosamina:albumina calculada para os grupo diestro $(9,53 \pm 2,2)$ e piometra $(11,43 \pm 2,6)$ aproximou-se da significância $(\mathrm{p}=0,08)$.

Frente à diferença significativa entre idade e peso das pacientes com piometra em comparação às pacientes em diestro, foi determinada a correlação linear de Pearson entre leucograma, idade e peso, contra os índices de sensibilidade à insulina calculados

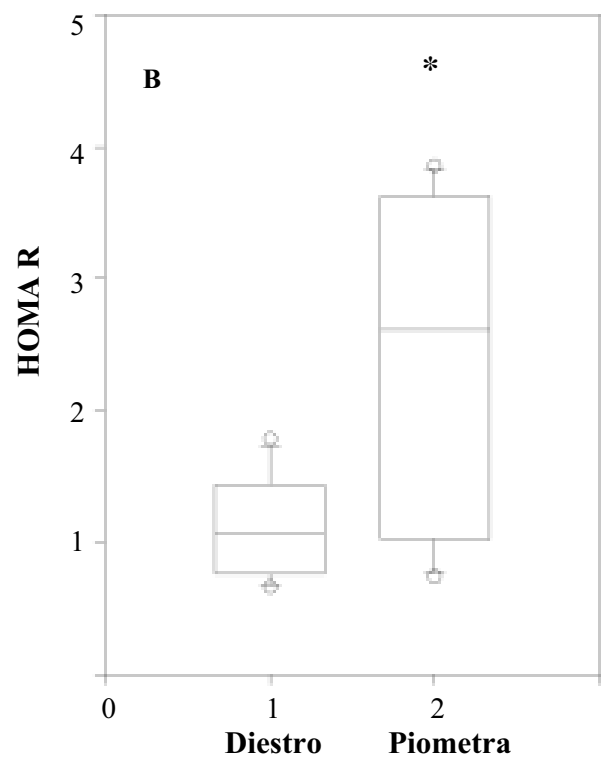

Figura 2. [A] Medianas, amplitude interquartil e percentis 10 e $90 \%$ do aumento no índice HOMA B em relação ao basal (grupo anestro = 1) dos grupos diestro e piometra. [B] Medianas, amplitude interquartil e percentis 10 e $90 \%$ do índice HOMA $\mathrm{R}$ dos grupos diestro e piometra. * representa diferença significativa $(\mathrm{p}<0,05)$ pelo teste de Mann-Whitney. 
Tabela 3. Média, desvio padrão e intervalo dos valores obtidos para fructosaminemia nas diferentes fases do ciclo estral e em pacientes com piometra.

\begin{tabular}{cc}
\hline Categoria & Fructosaminemia $(\boldsymbol{\mu m o l} / \mathbf{L}) *$ \\
\hline Anestro & $264,5 \pm 43,4(206,4-311,1)$ \\
Estro & $266,7 \pm 45,6(229,7-321)$ \\
Diestro & $287,2 \pm 56,3(250,9-423)$ \\
Piometra & $206,9 \pm 33,7(172,9-264,5)^{\#}$ \\
* valor de referência: 170 a $338 \mu \mathrm{mol} / \mathrm{L}[10] .{ }^{\#}$ representa diferença \\
significativa $(\mathrm{p}<0,05)$ entre o grupo piometra e o grupo diestro.
\end{tabular}

que obtiveram diferença significativa. A correlação entre os fatores de confusão leucograma, idade e peso também foi determinada. A Tabela 4 apresenta os valores de $r^{2}, r$ de Pearson e valor do $\mathrm{p}$ das correlações realizadas. O leucograma foi a variável que melhor se relacionou com os índices de sensibilidade à insulina, sendo significativa a correlação $(\mathrm{p}<0,05)$ para $\mathrm{HR}$, II e RI:GC. A variável peso só apresentou correlação com o HR; enquanto a variável idade apresentou correlação somente com o HB. Não foi observada correlação significativa entre leucograma, peso ou idade $(p>0,05)$.

\section{DISCUSSÃO}

Cerca de $70 \%$ dos casos de DMC ocorrem em fêmeas [11,13], sendo que na maioria dos casos observa-se que o início da doença está associado ao período de diestro [35]. Contudo, em uma série de casos norte-americanos, encontrou-se resistência à insulina secundária ao diestro em somente $8 \%$ dos casos totais $[9,19]$, provavelmente em decorrência da prática da castração ser mais rotineira naquele país. No Reino Unido, a incidência de DMC em fêmeas tem reduzido, apresentando uma distribuição igual entre machos e fêmeas, em decorrência da prática da esterilização precoce das fêmeas e menor uso de progestágenos sintéticos como anticoncepcionais $[4,19]$. Estudos dos receptores de insulina no tecido muscular esquelético de fêmeas caninas evidenciaram que a ocorrência das fases do ciclo estral perturba os mecanismos fisiológicos de sinalização da insulina. Além de uma menor atividade tirosina quinase de membrana, o que pode estar associado à menor ativação do receptor de insulina, o tecido muscular apresenta uma menor afinidade à insulina durante o estro e o diestro [2,39]. No entanto, demonstrou-se que o tecido muscular responde a esta menor sensibilidade,

Tabela 4. Resultados de $\mathrm{r}^{2}$, $\mathrm{r}$ de Pearson e valores de $\mathrm{p}$ das variáveis de sensibilidade à insulina correlacionadas aos fatores de confusão leucograma, idade e peso.

\begin{tabular}{|c|c|c|c|}
\hline Variáveis correlacionadas & $\mathbf{r}^{2}$ & r de Pearson & Valor de $p$ \\
\hline Leucograma $v s$. HOMA B & 0,073 & 0,272 & 0,346 \\
\hline Leucograma $v s$. HOMA R & 0,502 & 0,709 & $<0,01^{*}$ \\
\hline Leucograma $v s$. Índice insulinogênico & 0,421 & 0,649 & $0,012 *$ \\
\hline Leucograma $v s$. Ins/Gli corrigida & 0,381 & 0,618 & $0,0186^{*}$ \\
\hline Idade $v s$. HOMA B & 0,638 & 0,799 & $<0,001 *$ \\
\hline Idade $v s$. HOMA R & 0,244 & 0,494 & 0,072 \\
\hline Idade $v s$. Índice insulinogênico & 0,145 & 0,382 & 0,178 \\
\hline Idade vs. Relação Ins/Gli corrigida & 0,164 & 0,406 & 0,150 \\
\hline Peso vs. HOMA B & 0,142 & 0,378 & 0,183 \\
\hline Peso vs. HOMA R & 0,368 & 0,607 & $0,021^{*}$ \\
\hline Peso vs. Índice insulinogênico & 0,172 & 0,415 & 0,140 \\
\hline Peso vs. Relação Ins/Gli corrigida & 0,153 & 0,392 & 0,166 \\
\hline Peso vs. Leucograma & 0,235 & 0,485 & 0,078 \\
\hline Peso vs. Idade & 0,240 & 0,490 & 0,075 \\
\hline Idade $v s$. Leucograma & 0,104 & 0,324 & 0,258 \\
\hline
\end{tabular}

*representa diferença significativa $(\mathrm{p}<0,05)$ para análise de correlação de Pearson. 
com um aumento na capacidade total de ligação à insulina [39], motivo pelo qual não se observam alterações marcantes na glicemia durante um desafio com glicose em um teste de tolerância intravenoso [36].

Classicamente, a resistência à insulina observada durante o diestro é atribuída aos efeitos diretos da progesterona sobre a sensibilidade à insulina [45] e à liberação de hormônio do crescimento $(\mathrm{GH})$ pela glândula mamária estimulada pela progesterona [7,47]. Apesar disso, alguns autores consideram que o diestro per se não seria suficiente para promover diabetes em cães, mas que a progesterona poderia induzir a diabetes, especialmente se há outros fatores de risco associados [17,35,46], uma vez que a maior liberação de GH pela glândula mamária parece ser um fenômeno mais comum em cadelas mais velhas [9]. Além disso, alguns estudos demonstraram que a ocorrência das fases do ciclo estral promove prejuízos à secreção de insulina, inclusive com alterações na histologia pancreática [41,42]. Apesar disso, glicemia e insulinemia basais, e os diferentes índices de sensibilidade à insulina falharam em mostrar diferenças significativas entre as diferentes fases do ciclo estral.

$\mathrm{O}$ índice HOMA (homeostatic model assessment) de percentual de atividade das células $\beta$ (HOMA B) e de resistência à insulina (HOMA R) foi desenvolvido com objetivo de avaliar a sensibilidade à insulina $\mathrm{e}$ atividade das células $\beta$ utilizando somente os valores de glicemia e insulinemia em jejum; uma vez que a concentração de glicose e insulina em um estado basal é determinada por uma alça de retroalimentação [28]. Neste estudo, a aplicação do índice HOMA foi adaptada, a partir do seu uso em humanos. Como o estudo original considerou que pacientes humanos saudáveis, eutróficos e com menos de 35 anos apresentavam atividade das células $\beta$ de $100 \%$ e resistência à insulina $=1$, para fins de comparação, considerou-se que o grupo anestro (composto por cadelas com menos de 2 anos, saudáveis e com escore corporal ideal) apresentou atividade de células $\beta$ de $100 \%$ e resistência à insulina $=1$.

Apesar de não existirem valores de referência para os índices HOMA B e HOMA R para cães, esses índices são bem aceitos em medicina humana como indicadores de sensibilidade à insulina, já tendo inclusive sido validados frente a testes tidos como "padrão ouro" como o clamp normoglicêmico hiperinsulinêmico, apesar de se reconhecer o prejuízo da falta de um valor de corte estabelecido para classificação dos resultados dos pacientes humanos [16,21,34]. Mesmo sem ter apresentado diferenças significativas em relação aos grupos anestro e estro, a avaliação do índice insulinogênico e da relação insulina:glicose corrigida, mostrou valores acima dos limites de referência $[9,23]$ no grupo diestro, o que concorda com os inúmeros trabalhos que indicam a resistência à insulina durante o diestro $[2,7,19,35,47]$. Contudo, apesar de ter apresentado os valores mais altos no grupo diestro, a fructosamina, um excelente indicador de glicemia para as duas semanas anteriores à coleta [18,27], falhou em mostrar diferenças significativas entre as diferentes fases do ciclo estral.

A síndrome sepse representa um espectro de alterações patofisiológicas que resultam de uma resposta inflamatória exuberante pelo hospedeiro em resposta a um estímulo infeccioso ou não $[5,6]$. A inflamação apresenta um papel fundamental no processo de morte dos patógenos ao criar um ambiente hostil através da produção de moléculas oxidantes e ativação dos linfócitos B e T [14]. O quadro clínico da síndrome sepse pode variar de desarranjos fisiológicos moderados a severa disfunção múltipla de órgãos e morte, como pode ocorrer frente à piometra $[3,9,10,48]$. Um estudo evidenciou que $54 \%$ das pacientes com piometra avaliadas apresentavam pelo menos dois sinais cardinais de sepse; como leucocitose ou leucopenia, taquicardia, taquipneia e hipo ou hipertermia [1]. Todas as pacientes do grupo piometra do presente estudo apresentaram pelo menos dois desses sinais. Durante uma resposta inflamatória aguda, ocorre uma alteração metabólica de forma a permitir um maior aporte de nutrientes para o sistema imunológico (SI). Neste quesito, a presença de um estado de resistência à insulina moderado, promove uma menor captação de glicose pelo tecido muscular e adiposo, favorecendo a utilização da glicose pelo SI [43]. Citocinas próinflamatórias como a IL-1, IL-6, TNF- $\alpha$ medeiam o aumento nos lipídeos, estimulam a gliconeogênese e a produção de hormônios diabetogênicos e reduzem a sensibilidade à insulina [6,14].

O bloqueio à captação de glicose promovido pelo estado inflamatório tende a promover elevados valores de glicemia [5,6]. Maiores valores de hemoglobina glicosilada $(\mathrm{HbA} 1 \mathrm{c})$ também já foram detectados em pacientes humanos sofrendo de quadros inflamatórios crônicos [14]. Entretanto, a glicemia basal das 
pacientes com piometra, no presente estudo, não apresentou diferenças significativas em relação ao grupo diestro, ao contrário da insulinemia que fora significativamente superior, indicando um claro estado de resistência à insulina [9]. Contudo, o presente estudo observou uma fructosaminemia significativamente menor nas pacientes com piometra, quando comparadas às fêmeas em diestro. A explicação para este achado reside no fato de que a fructosamina deriva quimicamente das proteínas séricas, especialmente da albumina [18]. A concentração de fructosamina deve ser avaliada sempre que possível à luz da concentração plasmática total de proteínas e de albumina, uma vez que hipoproteinemia e hipoalbuminemia podem facilmente provocar valores reduzidos de fructosamina [18,26,27].

Apesar de, no presente estudo, não se ter detectado diferença significativa nos valores de proteína total determinados por refratometria (dados não apresentados) entre os grupos diestro e piometra, dois eventos podem explicar porque foi observado hipoalbuminemia e consequentemente menores valores de fructosamina nas pacientes com piometra: 1) frente à intensa reação inflamatória, espera-se um aumento na produção de globulinas, associado a uma redução na síntese de albumina com objetivo de manutenção do equilíbrio osmótico do líquido intravascular [20]. 2) O maior catabolismo protéico pode resultar em hipoalbuminemia, uma vez que a albumina é passível de servir como substrato energético frente a quadros catabólicos [49] e há um maior catabolismo em quadros sépticos frente à liberação de hormônios diabetogênicos [14], além do que, pacientes com piometra apresentam-se anoréxicos ou com hipofagia crônica $[9,48]$.

Apesar disso, os demais índices de sensibilidade à insulina calculados (II, RI:GC, HR e HB), foram eficazes em demonstrar um claro estado de resistência à insulina, como previamente identificado neste estudo, através da hiperinsulinemia, presente nas fêmeas com piometra. Estes resultados estão de acordo, e indicam de forma simples e direta a severa resistência muscular à insulina já previamente demonstrada por de estudos de ligação insulina-receptor e testes de tolerância à glicose em fêmeas caninas com piometra [31,38,39]. Frente a uma resistência à insulina mais severa como frente a um quadro séptico/inflamatório tal qual a piometra, ambos índice insulinogênico, relação insulina:glicose corrigida e o HOMA R mostraram-se bastante sensíveis na identificação de tal quadro metabólico. A ausência de valores de corte para o HOMA R dificulta sua interpretação [34], contudo a comparação com os valores do grupo diestro deixa clara a aplicabilidade deste índice adaptado da medicina humana, para identificação de quadros severos de resistência à insulina também em cães. Na mesma linha interpretativa, o índice HOMA B evidenciou convincentemente que esta resistência à insulina promovida pela piometra é acompanhada por uma maior atividade das células beta pancreáticas [28], resultando em maior insulinemia, como previamente demonstrado através da hiperinsulinemia basal presente nestas pacientes e em testes de tolerância à glicose, em que fềmeas com piometra apresentaram picos de insulinemia significativamente maiores do que fêmeas em outras fases do ciclo estral $[31,38]$. Esta maior atividade das células beta pancreáticas pode resultar em exaustão de células beta pancreáticas, levando à falência na homeostasia da glicose e ao início da diabetes [35]. Contudo, já se demonstrou que estados que provoquem hipersecreção de insulina podem não ser a causa de uma eventual exaustão celular, mas sim um fator desencadeante de destruição imunomediada das células beta $[9,17,32]$.

A determinação do escore corporal, conforme critérios pré-definidos [25], e adaptado neste trabalho a uma escala de 1 a 5 ( 1 = caquético, $5=$ obeso) foi importante no estudo de grupos homogêneos com relação à condição corporal, evitando assim possíveis diferenças na sensibilidade à insulina em decorrência de sobrepeso ou caquexia [9,29]. A maior predisposição à ocorrência de piometra em pacientes mais velhas, bem como em algumas raças identificadas no grupo piometra, como Rottweiller, Pastor Alemão e Collie, justificam a diferença significativa observada na idade e pesos das pacientes com piometra quando comparadas ao grupo diestro [33]. A diferença significativa na idade e no peso das pacientes do grupo piometra poderia representar fatores de confusão na interpretação dos resultados. Primeiramente, pode-se esperar uma menor sensibilidade à insulina em pacientes mais idosos [9] assim como em pacientes com pesos maiores, representando maior porte das pacientes, uma vez que para serem admitidas no estudo, um dos critérios de inclusão foi a presença de uma condição corporal eutrófica. Cães de grande porte apresentam maior secreção do hormônio diabetogênico GH durante o crescimento [8] e também maior concentração de somatotrofos na hipófise do que cães de menor porte [24]. 
Neste sentido, a associação moderada a forte da variável leucograma, com o índice insulinogênico, relação insulina:glicose corrigida e HOMA R evidenciou de forma clara que o estado de resistência à insulina observado nas pacientes com piometra deve-se especialmente à presença de um estado séptico/inflamatório marcado por uma leucometria elevada, e não à maior idade ou ao maior porte. Apesar disso, a variável peso associou-se positivamente com o HOMA $\mathrm{R}$, ao passo que a variável idade correlacionou-se positivamente com o índice HOMA B.

\section{CONCLUSÕES}

Os diferentes índices de sensibilidade à insulina estudados demonstraram sensibilidades diferentes na capacidade de indicar estados fisiológicos ou patológicos de resistência à insulina. Apesar disso, são índices simples, baratos e de fácil aplicação, apresentando vantagens em comparação a técnicas mais apuradas de estudos de sensibilidade à insulina (teste de tolerância à glicose oral ou intravenosa, teste de tolerância à insulina, clamp normoglicêmico hiperinsulinêmico, estudos de ligação hormônio-receptor). O cálculo de índices baseado em fórmulas simples baseadas na glicemia e insulinemia basais apresenta vantagens na avaliação de estados de resistência à insulina em comparação aos valores isolados de glicose e insulina, e de uma forma geral, quanto mais intensa a resistência à insulina, maior a sensibilidade dos diferentes índices. No entanto, a fructosamina não apresentou-se como um indicador sensível. Apesar de ser a avaliação menos onerosa em comparação à dosagem de glicemia e insulinemia, sua correlação com os valores de albuminemia a torna mais vulnerável a variações que interferem na detecção de uma situação de resistência à insulina. A relação fructosamina:albumina apresenta um bom potencial para resolver este problema, no entanto estudos posteriores fazem-se necessários para determinar valores de referência e pontos de corte para os índices HOMA R, HOMA B e relação fructosamina:albumina. Até o momento, os índices insulinogênico e relação insulina:glicose corrigida apresentam-se as melhores opções para avaliação da sensibilidade à insulina

\section{NOTAS INFORMATIVAS}

${ }^{1}$ CC-530, Celm, São Paulo, Brasil

${ }^{2}$ Accu-Check Active, Roche Diagnóstica, São Paulo, Brasil ${ }^{3}$ ImmuChem Coated Tube Insulin, MP Biomedicals, New York, U.S.A.

${ }^{4}$ Labtest Diagnóstica, Lagoa Santa, Brasil

\section{REFERÊNCIAS}

1 Basso P.C., Brun M.V., Barcellos H.H.A., Riviera F.B., Kreutz L.C., Anziliero D., Melatti L., Bairros M.C. \& Zilio P.P. 2006. Validação dos critérios de sepse em cães com piometra. In: Anais do XVII Congresso Estadual de Medicina Veterinária (Gramado, Brasil) 1CD-ROM.

2 Batista M.R., Smith M.S., Snead W.L., Connolly C.C., Lacy D.B. \& Moore M.C. 2005. Chronic estradiol and progesterone treatment in conscious dogs: effects on insulin sensitivity and response to hypoglicemia. American Journal of Physiology Regulatory Integrative and Comparative Physiology. 289: 1064-1073.

3 Blackwell T.S. \& Christman J.W. 1996. Sepsis and cytokines: current status. British Journal of Anaesthesia. 77: $110-117$.

4 Catchpole B., Ristic J.M., Fleeman L.M. \& Davison L.J. 2005. Canine diabetes mellitus: can old dogs teach us new tricks? Diabetologia. 48: 1948-1956.

5 Das U.N. 2003. Current advances in sepsis and septic shock with particular emphasis on the role of insulin. Medical Science Monitor. 9: RA181-192.

6 Das U.N. 2003. Insulin in sepsis and septic shock. Journal of the Association of Physicians from India. 51: 695-700.

7 Eingenmann J.E., Eingenmann R.Y., RijinberkA., Gaag I., Zapf J. \& Froesch E.R. 1983. Progesterona-controlled growth hormone overproduction and naturally occurring canine diabetes and acromegaly. Acta Endocrinologica. 104: $167-176$.

8 Favier R.P., Mol J.A., Kooistra H.S. \& Rijnberk, A. 2001. Large body size in the dog is associated with transient GH exsses at a young age. Journal of Endocrinology. 170: 479-484.

9 Feldman EC \& Nelson RW. 2004. Canine Diabetes Mellitus. In: Canine and Feline Endocrinology and Reproduction. 3rd. edn. Missouri: Saunders, pp.486-538.

10 Ferreira C.R. \& Lopes M.D. 2000. Complexo hiperplasia cística endometrial/piometra em cadelas - revisão. Clínica Veterinária. 27: 36-44.

11 Fleeman L.M. \& Rand J.S. 2001. Management of canine diabetes. Veterinary Clinics of North America: Small Animal Practice. 31: 855-879. 
12 Fransson B.A., Karlam E., Bergstrom A., Lagerstedt A.S., Park J.S., Evans M.A. \& Ragle C.A. 2004. C-reactive protein in the differentiation of pyometra from cystic endometrial hyperplasia/mucometra in dogs. Journal of the American Animal Hospital Association. 40: 391-399.

13 Greco D.S. 2001. Diagnosis of diabetes mellitus in dogs. Veterinary Clinics of North America: Small Animal Practice. 31 : 844-853.

14 Grimble R.F. 2002. Inflammatory status and insulin resistance. Current Opinion in Clinical Nutrition and Metabolic Care. 5: 551-559.

15 Guptill L., Glickman L. \& Glickman N. 2003. Time trends and risk factors for diabetes mellitus in dogs: analysis of veterinary medical data base records. The Veterinary Journal. 165: 240-247.

16 Gutt M., Davis C.L., Spitzer S.B., Llabre M.M., Kumar M., Czarnecki E.M., Schneiderman N., Skyler J.S. \& Marks J.B. 2000. Validation of the insulin sensitivity index (ISI0,120): comparison with other measures. Diabetes Research and Clinical Practice. 47: 177-184.

17 Hoenig M. 2002. Comparative aspects of diabetes mellitus in dogs and cats. Molecular and Cellular Endocrinology. 197: 221-229.

18 Jensen A.L. 1995. Gycated blood proteins in canine diabetes mellitus. Veterinary Record. 137: 401-405.

19 Johnson C.A. 2008. Glucose homeostasiss during canine pregnancy: Insulin resistance, ketosis, and hypoglycemia. Theriogenology. 70: 1418-1423.

20 Kaneko J.J. 1997. Carbohydrate Metabolism and Its Diseases. In: Kaneko J.J., Harvey J.W. \& Bruss M.C. (Eds). Clinical Biochemistry of Domestic Animals. 5th. edn. Missouri: Academic Press, 932 p.

21 Katz A., Nambi S.S., Mather K., Baron A.D., Follmann D.A., Sullivan G. \& Quon M.J. 2000. Quantitative insulin sensitivity check index: a simple, accurate method for assessing insulina sensitivity in humans. The Journal of Clinical Endocrinology \& Metabolsim. 85: 2402-2410.

22 Klinkenberg H., Sallander M.H. \& Hedhammar A. 2006. Feeding, exercise and weight identified as risk factors in canine diabetes mellitus. Journal of Nutrition. 136: 1985S-1987S.

23 Kogika M.M., Brandão L.P., Jericó M.M., Hagiwara M.K., Simões D.M.N. \& Mendonça B. 2001. Determinação das concentrações séricas de glicose e insulina de cães em choque endotóxico. Ciência Rural. 31: 813-817.

24 Krook L., Larsson S. \& Rooney J.R. 1960. The interrelationship of diabetes mellitus, obesity and pyometra in the dog. American Journal of Veterinary Research. 1: 120-124.

25 Laflame D.P. 1997. Development and validation of a body condition score system for dogs. Canine Practice. 22: 10-15.

26 Loste A. \& Marca M.C. 1999. Study of the effect of total serum protein and albumin concentrations on canine fructosamine concentration. Canadian Journal of Veterinary Research. 63: 138-141.

27 Loste A. \& Marca M.C. 2001. Fructosamine and glycated hemoglobin in the assesment of glycaemic control in dogs. Veterinary Research. 32: 55-62.

28 Matthews D., Hosker J.P., Rudenski A.S., Naylor B.A., Treacher D.F. \& Turner R.C. 1985. Homeostasis model assessment: insulin resistance and $\beta$-cell function from fasting glucose and insulina concentrations in man. Diabetologia. 28: $412-419$.

29 Matthews D., Rottiers M.D., Kaneko J.J. \& Vermeulen M.D. 1984. Diabetes mellitus in dogs: relationship of obesity to glucose tolerance and insulin response. American Journal of Veterinary Research. 45: 98-103.

30 Neuvians T.P. \& Berger M. 2002. Diabetes care in cats and dogs. Diabetic Medicine. 19: 77-79.

31 Neves K.S., Pöppl A.G., Souza F.E., Koenen J.V., Mottin T.S., Días C.B., Da Silva R.S.M. \& Kucharski L.C. 2006. Avaliação da tolerância à glicose em fêmeas caninas durante o ciclo estral e na condição hiperplasia endometrial cística piometra. In: Anais do XVII Congresso Estadual de Medicina Veterinária (Gramado, Brasil) -1 CD-ROM.

32 Nilsson C., Ursing D., Törn C., Aberg A. \& Landin - Olsson M. 2007. Presence of GAD antibodies during gestacional diabetes mellitus predicts type 1 diabetes. Diabetes Care. 30: 1968-1971.

33 Niskanen M. \& Thrusfield M.V. 1998. Associations between age, parity, hormonal therapy and breed, and pyometra in Finnish dogs. The Veterinary Record. 143: 493-498.

34 Oliveira E.P., Souza M.L.A. \& Lima M.D.A. 2005. Índice HOMA (homeostasis model assessment) na prática clínica: uma revisão. Jornal Brasileiro de Patologia e Medicina Laboratorial. 41: 237-243.

35 Pöppl A.G. \& González F.H.D. 2005. Aspectos epidemiológicos e clínico-laboratoriais da diabetes mellitus em cães. Acta Scientiae Veterinarie. 33: 33-40. 
36 Pöppl A.G., Mottin T.S., Dias C.B., Breitsmater I., Beck C.A.C., Lasta C., González F.H.D., Kucharski L.C. \& Da Silva R.S.M. 2007. Estudo preliminar da ligação hormônio-receptor da insulina em membranas de músculo e da tolerância à glicose em fêmeas caninas durante o ciclo estral. Acta Scientiae Veterinariae. 35: 462-464.

37 Pöppl A.G., Muccillo M.S., Neuwald E.B., Sortica M.S., Cheuiche S., Lamberts M. \& Gomes C. 2005. Diabetes mellitus hiperosmolar não cetósico em uma cadela com piometra - relato de caso. Revista da Universidade Rural - Ser Ciências Vida. 25 (Supl.): 193-194.

38 Pöppl A.G., Souza F.E., Neves K.S., Beck C.A.C., Leal J.S., Driemeier D., Lasta C.S., González F.H.D., Valle S.C., Kucharski L.C. \& Da Silva R.S.M. 2007. Influência do complexo hiperplasia endometrial cística - piometra na sensibilidade periférica à insulina e predisposição à diabetes mellitus: resultados preliminares. Acta Scientiae Veterinariae. 35(Supl. 2): s465-s467.

39 Pöppl A.G., Valle S.C., Beck C.A.C., Gonzalez F.H.D, Kucharski L.C. \& Da Silva, R.S.M. 2009. Effect of estrus cycle and pyometra on insulin receptor tyrosine quinase activity and insulin receptor binding in female dogs. Clínica Veterinária. 14 (Supl.): 136-138.

40 Rand J.S., Fleeman L.M., Farrow H.A., Appleton D.J. \& Lederer R. 2004. Canine and feline diabetes mellitus: nature or nurture? Journal of Nutrition. 134: 2072s-2080s.

41 Renauld A., Gomez N.V., Scaramal J.D., Garrido D. \& Wanke M.M. 2003. Serum insulin, glucose and non esterified fatty acids after administration of follicle-stimulating and luteinizing hormones in bitches. Medicina (Buenos Aires). 63: 28-32.

42 Renauld A., Sverdlik R.C., Lawzewitsch I.V., Agüero A., Pérez R.L., Rodríguez R.R. \& Foglia V.G. 1987. Metabolic and histologycal pancreatic changes induced by ovariectomy in the female dog. Acta Physiologica Pharmacologica Latinoamericana. 37: 289-304.

43 Rey A.D., Roggero E., Randolf A., Mahuad C., McCann S., Rettori V. \& Besedovsky, H.O. 2006. IL-1 resets glucose homeostasis at central levels. PNAS. 103(43): 16039-16044.

44 Rijnberk A., Kooistra H.S. \& Mol J.A. 2003. Endocrine diseases in dogs and cats: similarities and differences with endocrine diseases in humans. Growth Hormone and IGF Reasearch. 13: S158-S164.

45 Ryan E.A. \& Enns L. 1988. Role of gestacional hormones in the induction of insulin resistance. Journal of Clinical Endocrionology and Metabolism. 67: 341.

46 Scaramal J.D., Renauld A., Gomez N.V., Garrido D., Wanke M.M. \& MarquezA.G. 1997. Natural estrous cycle in normal and diabetic bitches in relation to glucose and insulin tests. Medicina (Buenos Aires). 57: 169-180.

47 Selman P.J., Mol J.A., Rutteman G.R. \& Rijnberk A. 1994. Progestin treatment in the dog I. Effects on growth hormone, insuli-like growth factorI and glucose homeostasis. European Journal of Endocrinology. 131: 413-421.

48 Smith F.O. 2006. Canine pyometra. Theriogenology. 66: 610-612.

49 Thrall M.A. 2004. Erythrocyte Morphology. In: Thrall M.A. (Ed). Veterinary Hematology and Clinical Biochemistry. Maryland: Lippincott Williams \& Wilkins, 518p.

50 Vannucchi C.I., Satzinger S. \& Santos S.E.C. 1997. Técnica de citologia vaginal como método de diagnóstico da fase do ciclo estral em cadelas. Clínica Veterinária. 9: 14-19.

51 Wagner M.B. 1998. Aspectos básicos da sumarização de informações em medicina. Jornal de Pediatria. 74: 71-76. 